\title{
SELF-ASSEMBLED LIPOSOME-LOADED MICROBUBBLES: THE MISSING LINK FOR SAFE AND EFFICIENT ULTRASOUND TRIGGERED DRUG-DELIVERY
}

Bart Geers ${ }^{1}$, Ine Lentacker ${ }^{1}$, Niek N. Sanders ${ }^{2}$, Joseph Demeester ${ }^{1}$, Stephen Meairs $^{3}$, Stefaan C. De Smedt ${ }^{1 *}$

${ }^{1}$ Ghent Research Group on Nanomedicines, Lab of General Biochemistry and Physical Pharmacy, Faculty of Pharmaceutical Sciences, Ghent University, Harelbekestraat 72, 9000 Gent, Belgium

${ }^{2}$ Laboratory of Gene Therapy, Faculty of Veterinary Medicine, Ghent University, Heidestraat 19, 9820 Merelbeke, Belgium

${ }^{3}$ Department of Neurology, Universitätsklinikum Mannheim, Heidelberg University, Theodor-Kutzer-Ufer 1-3, 66167 Mannheim, Germany

*Corresponding author: Laboratory of General Biochemistry and Physical Pharmacy, Harelbekestraat 72, 9000 Gent, Belgium, Tel: +32 926480 76, Fax: +32 92648189 stefaan.desmedt@ugent.be 


\section{INTRODUCTION}

Microbubbles are gas filled microspheres which are approved for diagnostic ultrasound contrast imaging by the FDA. Such bubbles, a few microns in size, are usually filled with an hydrophobic gas and are stabilized by a surfactant (lipid, protein or polymer) shell, to enhance their shelf life and circulation time in blood after injection. Because of the difference in density between the gas core of the microbubble and the surrounding fluid, microbubbles start to oscillate when subjected to high frequency $(1-10 \mathrm{MHz})$ ultrasound. This "cavitation" of microbubbles has been intensively studied by means of high speed optical imaging [1-3] and can be divided into respectively stable and inertial cavitation. In an ultrasound field with a low acoustical pressure microbubbles are stably cavitating and will oscillate around a given diameter. Inertial cavitation on the other hand occurs at higher acoustical pressures, the movement of the microbubbles becomes more violent which leads to destruction of the microbubbles [4]. This microbubble destruction produces distinct nonlinear acoustic echoes which are very useful in contrast enhanced ultrasound imaging [5]. Cavitation of microbubbles may also induce biological and mechanical effects on the surrounding space which may be particularly useful for drug delivery [6].

When microbubble collapse occurs in the vicinity of cells it has been shown that plasma membranes are temporarily permeabilized through the formation of transient pores in the cellular membrane, induced by micro-jets and shockwaves produced after microbubble collapse [3,7]. This phenomenon is called sonoporation [6]. It is believed that sonoporation is strongly dependent on the acoustical properties of the applied ultrasound field $[8,9]$. Such transient pores also enhance the uptake of macromolecules in cells [10]. Recent studies show that sonoporation effects may last 
up to 24 hours after ultrasound treatment [11]. The use of microbubbles in combination with ultrasound may even induce openings in the blood-brain barrier which could be of interest to tackle drug delivery into the brain which currently remains a huge challenge $[12,13]$.

Only if the drug molecules are physically located in the vicinity of microbubbles subjected to ultrasound, it is expected that sonoporation will enhance drug uptake by cells. Moreover, as these effects will only occur where and when ultrasound is applied, drug delivery is expected only to happen in the insonated tissue. Hence, for ultrasound targeted drug delivery it is crucial to design microbubbles which can be loaded with drug molecules. Meanwhile a number of concepts for drug loaded microbubbles are under investigation [14-16]. Basically, microbubbles can be loaded with drugs in three ways: (a) the drug can be incorporated in the microbubble shell [17], (b) (lipophilic) drugs can be incorporated in an inner oil phase present in the microbubbles [18] or (c) "colloidally drug loaded microbubbles" can be obtained through the attachement of drug containing nanoparticles (like e.g. liposomes) on the microbubbles' surface as reviewed by Bohmer et al. and Lentacker et al $[19,20]$. Independent on the way the microbubbles become loaded with drugs, they should fulfill at least the following requirements: (a) the microbubbles should be loaded with a sufficient amount of drug; (b) the shelf-life of the drug-loaded microbubbles should be long enough and (c) the drug-loaded microbubbles should be designed without the incorporation of toxic or immunogenic substances. We believe that loading the surface of microbubbles with drug containing liposomes is a promising concept for ultrasound guided drug delivery as: (a)more drugs can be loaded on microbubbles compared with other loading strategies, (b) a plethora of knowledge is available on liposomes for drug delivery which can be perfectly used to develop the colloidally 
loaded microbubble concept and (c) importantly, certain liposomes are safe and even already used in clinical practice.

Recently we succeeded in preparing respectively doxorubicin (DOX)-liposome loaded microbubbles [21] and lipoplex-loaded microbubbles (containing pDNA or siRNA). We showed that in combination with ultrasound, such microbubbles strongly improved both doxorubicin (DOX) cytotoxicity and pDNA [16,22] and siRNA [23] delivery to cells in vitro. However, the complex microbubble preparation method, the immunogenic nature of the avidin-biotin chemistry used to link the liposomes/lipoplexes to the microbubbles and the successive washing steps, made this material not ideal for easy clinical use. It is clear that the concept of liposome/lipoplex-loaded microbubbles needs further development and improvement. In this work we faced the challenge to design DOX-liposome loaded lipid shelled microbubbles through the self-assembly of the involved compounds. Importantly, the method we propose involves just a single step and allows to make a sterile material. As illustrated in Figure 1 and further explained in the results section, to a mixture of phospholipids, so named "functionalized DOX-liposomes" (i.e. DOX-liposomes containing MALEIMIDE functionalized PEG-lipids) and a hydrophobic gas were added. We show that under appropriate conditions these compounds self-assemble into DOX-liposome loaded microbubbles which are responsive to ultrasound and efficiently kill cells. 


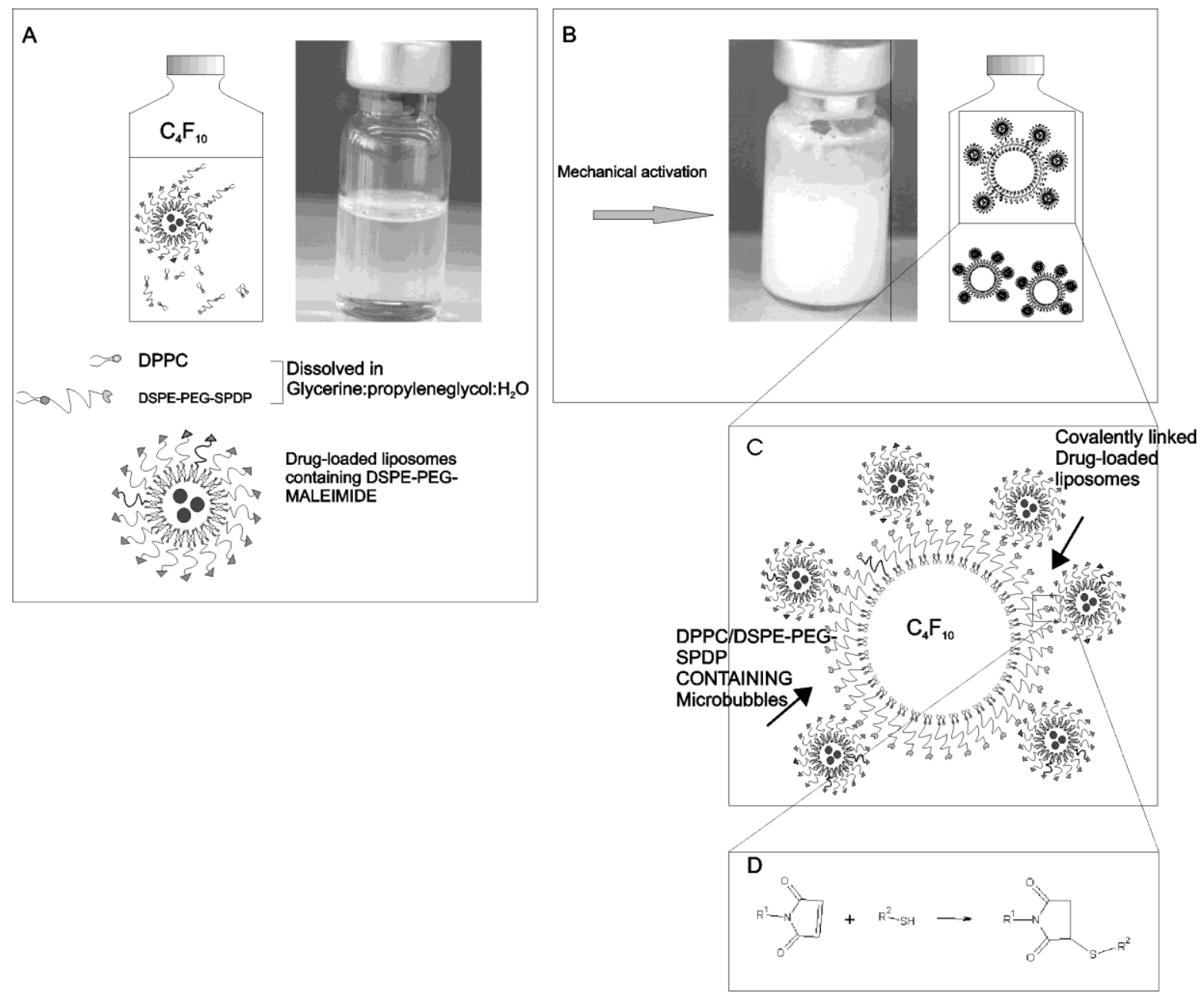

Figure 1: Schematic depiction of the preparation of liposome-loaded through self-assembly of the different constituents: Drug loaded liposomes containing DSPE-PEG-MALEIMIDE and perfluorobutane $\left(\mathrm{C}_{4} \mathrm{~F}_{10}\right)$ gas were added to vials containing: DPPC and DSPE-PEG-SPDP dissolved in a glycerin:propyleneglycol: $\mathrm{H}_{2} \mathrm{O}$ mixture (A) and were mechanically activated using a Capmix $^{\mathrm{TM}}$ device. This mechanical activation gives rise to lipid-shelled microbubbles (B) loaded with liposomes. These microbubbles are filled with the hydrophobic $\mathrm{C}_{4} \mathrm{~F}_{10}$ gas (C). The liposomes become coupled to the microbubbles' surface through covalent thiol-maleimide linkages (D). Note that the microbubbles are a few microns in size while the liposomes are some hundreds of nanometers. 


\section{MATERIALS AND METHODS}

\subsection{Preparation and characterization of functionalized microbubbles}

"Functionalized microbubbles" (i.e. microbubbles containing thiol-functionalized PEG-lipids) were prepared starting from a lipid solution being a mixture of 1,2dipalmitoyl-sn-glycero-3-phosphocholine (DPPC) and 1,2-distearoyl-sn-glycero-3phosphoethanolamine-N-[PDP(polyethylene glycol)-2000] (DSPE-PEG-PDP) (Avanti polar lipids, Albaster, AL) in a 1:2:7 glycerine-propyleneglycol- $\mathrm{H}_{2} \mathrm{O}$ solvent (Sigmaaldrich,Bornem,Belgium); the molar ratio of the lipids in the lipid solutions was respectively 97:3, 92:8 and 65:35. The lipid solution was prepared as follows. Aliquots of both lipids, dissolved in $\mathrm{CHCL}_{3}$, were transferred into a round bottom flask. After $\mathrm{CHCl}_{3}$ evaporation, the lipid film was dissolved in a 1:2:7 glycerinepropyleneglycol- $\mathrm{H}_{2} \mathrm{O}$ mixture to obtain a clear solution with a final lipid concentration of $4 \times 10^{-4} \mathrm{mmol} / \mathrm{ml}$ (or $2 \times 10^{-4} \mathrm{mmol} / \mathrm{ml}$ for microbubbles used in the cell experiments). Aliquots of this lipid solution were transferred into $2.5 \mathrm{ml}$ chromatography vials, which headspace was filled with $\mathrm{C}_{4} \mathrm{~F}_{10}$ gas (F2 chemicals, Preston, UK). Finally, functionalized microbubbles (with an average size of $3 \mu \mathrm{m}$ ) were obtained by high speed shaking of the lipid solution in a Capmix ${ }^{\mathrm{TM}}$ device (3MESPE, Diegem, Belgium) during $15 \mathrm{sec}$. The size and the concentration of the microbubbles in the dispersion (i.e. number of microbubbles per $\mathrm{mL}$ ) were determined with a Beckman-coulter Multisizer 4 (Beckman-coulter, Brea, CA)

\subsection{Preparation of non-functionalized microbubbles}

Preparation of so named "non-functionalized microbubbles" occurred as described above for the functionalized bubbles, however 1,2-distearoyl-sn-glycero-3- 
phosphoethanolamine-N-[methoxy(polyethylene glycol)-2000 (DSPE-MPEG) (Avanti polar lipids, Albaster, AL) was used as a PEG-lipid instead of DSPE-PEG-PDP.

\subsection{Preparation and characterization of bodipy-labeled and DOX-liposomes}

Bodipy-labeled liposomes were prepared by transferring DPPC, DSPE-PEGMALEIMIDE) (Avanti polar lipids, Albaster, AL), cholesterol (Sigma-alldrich, Bornem, Belgium) and cholesteryl-bodipy (Invitrogen, Merelbeke, Belgium), all dissolved in $\mathrm{CHCl}_{3}$, in a 49:15:35:1 molar ratio to a round bottom flask. After $\mathrm{CHCl}_{3}$ evaporation, the remaining lipid film was rehydrated with HEPES buffer $(50 \mathrm{mM}, \mathrm{pH} 7.4$, Sigmaalldrich, Bornem, Belgium) to obtain a liposomal dispersion with a final lipid concentration of $16 \mathrm{mg} / \mathrm{ml}$. The liposomal dispersion was hereafter extruded through a $200 \mathrm{~nm}$ filter using a mini-extruder at $60^{\circ} \mathrm{C}$ (Avanti polar lipids, Albaster, AL).

DOX-liposomes were prepared as described by lentacker et al [21]. Briefly, DPPC, DSPE-PEG-MALEIMIDE and cholesterol dissolved in $\mathrm{CHCl}_{3}$ at a concentration of $20 \mathrm{mg} / \mathrm{ml}$ and $10 \mathrm{mg} / \mathrm{ml}$ respectively, were transferred in a round bottom flask in a 49:15:36 molar ratio. After evaporation the remaining lipid film was rehydrated with ammonium sulfate buffer $(250 \mathrm{mM})$ to obtain a liposomal dispersion with a final lipid concentration of $16 \mathrm{mg} / \mathrm{ml}$. The resulting liposome dispersion was extruded through a $200 \mathrm{~nm}$ filter using a mini-extruder at $60^{\circ} \mathrm{C}$. After extrusion the excess of ammonium sulfate was removed by overnight dialysis against distilled water (MWCO: 8000 Da, Spectra/Por Biotech, Compton, CA). Hereafter the liposomal dispersion was aliquoted into $450 \mu \mathrm{l}$ aliquots and liposomes were loaded with $\mathrm{DOX}$ by adding $50 \mu \mathrm{l}$ of a doxorubicin. $\mathrm{HCl}$ (Sigma-alldrich, Bornem, Belgium) 10 $\mathrm{mg} / \mathrm{ml}$ solution. Non-encapsulated DOX was removed in a second overnight dialysis against distilled water. Final DOX concentration was evaluated using absorbance 
spectrometry using an Envision plate-reader and reached between 0.5 and $0.8 \mathrm{mg} / \mathrm{ml}$ (Perkin-elmer, Waltham, MA)

\subsection{Preparation of liposome-loaded microbubbles}

Liposome-loaded microbubbles were prepared by adding a certain volume of the liposome dispersion to the lipid solution used for the preparation of microbubbles (see above, under 1.1 and 1.2). Liposome-loaded microbubbles were obtained by high-speed shaking of this liposome/lipid mixture in a Capmix ${ }^{\mathrm{TM}}$ device. The loading of the microbubbles with (bodipy-containing) liposomes was visualized using a Nikon EZC1 confocal microscope equipped with a 60x lens imaging using the $490 \mathrm{~nm}$ excitation light, while fluorescence was detected at $551 \mathrm{~nm}$.

\subsection{Evaluation of the loading of the microbubbles with liposomes by flow cytometry}

How different parameters influence the loading of microbubbles with bodipylabeled liposomes was determined using a flow cytometer (BD FACScalibur, Erembodegem, Belgium). Herefore $300 \mu \mathrm{l}$ of (bodipy-) liposome-loaded microbubbles were diluted in $300 \mu \mathrm{l}$ of HEPES buffer. The $488 \mathrm{~nm}$ laser of the flow cytometer was used to excite the bubbles while the emitted fluorescence was detected in the 530 $\mathrm{nm}$ channel FL1. Results were expressed as mean fluorescence per microbubble using unloaded microbubbles as a blank. For measurements on microbubbles in plasma, platelet poor plasma was obtained by centrifuging whole blood samples first at $300 \mathrm{~g}$ for $10 \mathrm{~min}$ and subsequently at $3200 \mathrm{~g}$ for $10 \mathrm{~min}$. $20 \mu \mathrm{l}$ of (bodipy-) liposome-loaded microbubbles were suspended in $150 \mu \mathrm{l}$ of plasma and diluted with $200 \mu \mathrm{l} \mathrm{Hepes} \mathrm{buffer.} \mathrm{All} \mathrm{the} \mathrm{flow} \mathrm{cytometry} \mathrm{experiments} \mathrm{were} \mathrm{performed} \mathrm{in} \mathrm{triplicate}$ and the presented data are the mean values. 


\subsection{Evaluation of the loading of the microbubbles with liposomes by Coulter Counter measurements}

The loading of the microbubbles with liposomes was further studied by the electrical sensing zone method with a Beckman-Coulter Multisizer 4 Coulter Counter. For each Coulter Counter experiment $20 \mu \mathrm{l}$ of (liposome-loaded) microbubbles were diluted in $10 \mathrm{ml}$ of Phosphate Buffered Saline (PBS, GIBCO, Merelbele, Belgium); 50 $\mu \mathrm{l}$ of this dilution was applied in the Multisizer 4 which was equipped with a $20 \mu \mathrm{m}$ aperture tube. The liposome-loaded microbubbles used in the Coulter Counter experiments were obtained by transferring $1 \mathrm{ml}$ of lipid solution (lipid concentration: 4 $\times 10^{-4} \mathrm{mmol} / \mathrm{ml}$ ) and respectively $0,35,100$ and $200 \mu \mathrm{l}$ bodipy liposomes into a $2.5 \mathrm{ml}$ chromatography vial, followed by high-speed shaking in the Capmix ${ }^{\mathrm{TM}}$ device.

We determined respectively the number of (unloaded and liposome-loaded) microbubbles per $\mathrm{mL}$ and the volume of all bubbles present in $1 \mathrm{ml}$ of dispersion (i.e. "total volume per ml"). The volume of a single microbubble could then be determined by equation (1).

(1) volume per bubble $=\frac{\text { total volume per } \mathrm{ml}}{\text { number of bubbles per } m l}$

The volume increase of a microbubble upon loading with lipsosomes (i.e. "liposome volume per bubble") could be calculated by equation (2) below.

(2) liposome volume per bubble $=$ volume of a loaded bubble - volume of an unloaded bubble,

Dividing this liposome volume per microbubble by the volume of a single (spherical) liposome (with an average diameter of approximately $200 \mathrm{~nm}$ as was 
determined by dynamic light scattering) gives an estimation of the amount of liposomes loaded on one microbubble (equation 3).

(3) number of liposomes per bubble $=\frac{\text { liposome volume per bubble (2) }}{\text { volume of one liposome }}$

All measurements were performed at least in triplicate and results are presented as the mean of three different measurements.

\subsection{Ultrasound induced DOX-release}

The release of doxorubicin from the liposomes was determined by fluorescence measurements. The following samples were prepared for the release experiments. One $\mathrm{ml}$ of lipid solution was mixed with 10 or $20 \mu \mathrm{l}$ of DOX-liposomes with a DOXconcentration of $0.1 \mathrm{mg} / \mathrm{ml}$ and $\mathrm{C}_{4} \mathrm{~F}_{10}$ gas. After shaking the lipid/lipsome mixture in the Capmix ${ }^{\mathrm{TM}}$ device the mixture was diluted with PBS to a final volume of $10 \mathrm{ml}$ and injected in an Opticell ${ }^{\mathrm{TM}}$ (Biocrystal, Westerville, $\mathrm{OH}$ ) plate. This plate was submerged in a water bath $\left(37^{\circ} \mathrm{C}\right)$ with an absorbing rubber and subjected to ultrasound during $15 \mathrm{~s}$ using the Sonitron device (Artison Corporation, Inola, OK, USA) equipped with a $2 \mathrm{~cm}$ ultrasound probe[22,24]. This probe was used with an ultrasound frequency of $1 \mathrm{MHz}$ with $20 \%$ duty cycle at an ultrasound intensity of 2 $\mathrm{W} / \mathrm{cm}^{2}$. Subsequently, the fluorescence of each sample was measured in a Wallac envision plate reader $\left(\lambda_{\mathrm{exc}} 500 \mathrm{~nm}, \lambda_{\mathrm{em}} 550 \mathrm{~nm}\right)$. Each experiment was performed at least in triplicate.

\subsection{Cell culture and cytotoxicity tests}

BLM cells [24] (melanoma cells) were cultured in F12-supplemented Dulbecco's modified Eagles medium (DMEM-F12) which contained 1\% Penicilin/Streptomycin, 2mmol/l glutamine, $10 \%$ Fetal bovine serum (FBS) (all purchased from Gibco, 
Merelbeke, Belgium) and $100 \mathrm{mmol} / \mathrm{l}$ HEPES $\mathrm{pH}$ 7.2. Cells were grown in an humidified incubator at $37^{\circ} \mathrm{C}$ in a $5 \% \mathrm{CO}_{2}$ atmosphere.

BLM cells were seeded in Opticell ${ }^{\mathrm{TM}}$ plates $\left(1.3 \times 10^{6}\right.$ cells per Opticell $\left.{ }^{\mathrm{TM}}\right)$ and were grown to $90 \%$ confluency, which was reached two days after seeding. Before experiments were performed, cells were washed with PBS. Subsequently, DOXliposome loaded microbubbles were added to the cells. The following samples were prepared for the cytotoxicity experiments. To $500 \mu \mathrm{l}$ of lipid solution (lipid concentration of $2 \times 10^{-4} \mathrm{mmol} / \mathrm{ml}$ ) respectively $10,100,250$ or $500 \mu \mathrm{l}$ of DOX liposomes (with a DOX concentration of $0.54 \mathrm{mg} / \mathrm{ml}$ ) were added; HEPES-buffer (50 $\mathrm{mM}, \mathrm{pH}$ 7.4) was added until a final volume of $1 \mathrm{ml}$ was reached. Before shaking vials were filled with $\mathrm{C}_{4} \mathrm{~F}_{10}$ gas. After shaking in the Capmix ${ }^{\mathrm{TM}}$ device, the $1 \mathrm{ml}$ microbubble dispersion was added to $10 \mathrm{ml}$ of optimem and this mixture was added to the cells in Opticell ${ }^{\mathrm{TM}}$ plates. Subsequently, these plates were submerged in a warm water bath $\left(37^{\circ} \mathrm{C}\right)$ with a bottom of ultrasound absorbing rubber. Ultrasound was delivered by moving the Sonitron ultrasound probe over the whole plate during 10-15 sec. We used $1 \mathrm{MHz}$ ultrasound with a $20 \%$ duty cycle with an ultrasound intensity of $2 \mathrm{~W} / \mathrm{cm}^{2}$. During ultrasound application cells were located on the top membrane of the Opticell ${ }^{\mathrm{TM}}$, in this way microbubbles are directly contacting the cells during sonication. After ultrasound application cell displacement was evaluated by means of microscopy and was always minimal with the microbubble concentrations used for these experiments.

After 4 hours of incubation, microbubbles were removed, cells were washed with PBS and incubated in fresh culture medium. After 24 hours cells were washed and the MTT reagent (Cell proliferation kit I, Roche diagnostics, Leuven, Belgium) was added for 4 hours. Subsequently, the sollubilization reagent was added and cells 
were incubated overnight to allow cell lysis at $37^{\circ} \mathrm{C}$. The next day, the absorbance of each plate was measured in an absorbance plate reader at respectively $590 \mathrm{~nm}$ $\left(\mathrm{OD}_{590}\right)$ to determine the formed formazan and at $690 \mathrm{~nm}\left(\mathrm{OD}_{690}\right)$ as a reference. The results of the cytotoxicity measurements are expressed as percentages; the viability of the cells which were only treated with optimem was considered to be $100 \%$, while the viability of cells exposed to phenol was considered to be $0 \%$. Experiments were performed at least in triplicate.

\subsection{Statistical analysis}

All data are presented as means $+/$ - one standard deviation. A student's t-test was performed to determine whether datasets differed significantly. A p-value smaller than 0.05 was regarded significant.

\section{RESULTS}

\subsection{Preparation of liposome-loaded microbubbles by self-assembly}

As schematically presented in Figure 1, to make liposome-loaded microbubbles, to a mixture of the (functionalized) lipids DPPC and DSPE-PEGSPDP, dissolved in a glycerine-propyleneglycol-water solvent at a concentration below their critical micellar concentration [25], liposomes containing DSPE-PEGMALEIMIDE were added. We observed that liposomes dispersed in the lipid solution are stable and can be stored during at least several weeks (data not shown). Therefore we stored the liposome/lipid dispersion at $4^{\circ} \mathrm{C}$ in chromatography vials. To "activate" the mixture, which refers to (as formation of liposome loaded microbubbles (Figure 1C), the vials' headspaces were filled with perfluorobutane gas and subsequently mixed with a high speed shaking-device (Capmix ${ }^{\mathrm{TM}}$ or Vialmix $\left.{ }^{\mathrm{TM}}\right)$. The Capmix $^{\mathrm{TM}}$ disperses the lipophilic perfluorobutane in the lipid solution. Hence, the 
hydrophobic tales of the dissolved lipids interact with the dispersed gas and stabilize the gas bubbles formed. Because functionalized (SPDP-) PEG-lipids were used which can interact with the functionalized (MALEIMIDE-)PEG-lipids of the liposomes (Figure 1D), liposome-loaded microbubbles were spontaneously formed during this process as can be seen in the confocal image in Figure $2 \mathrm{~B}$ and its corresponding transmission image (Figure 2A) clearly show bodipy-labeled liposomes at the surface of the microbubbles.
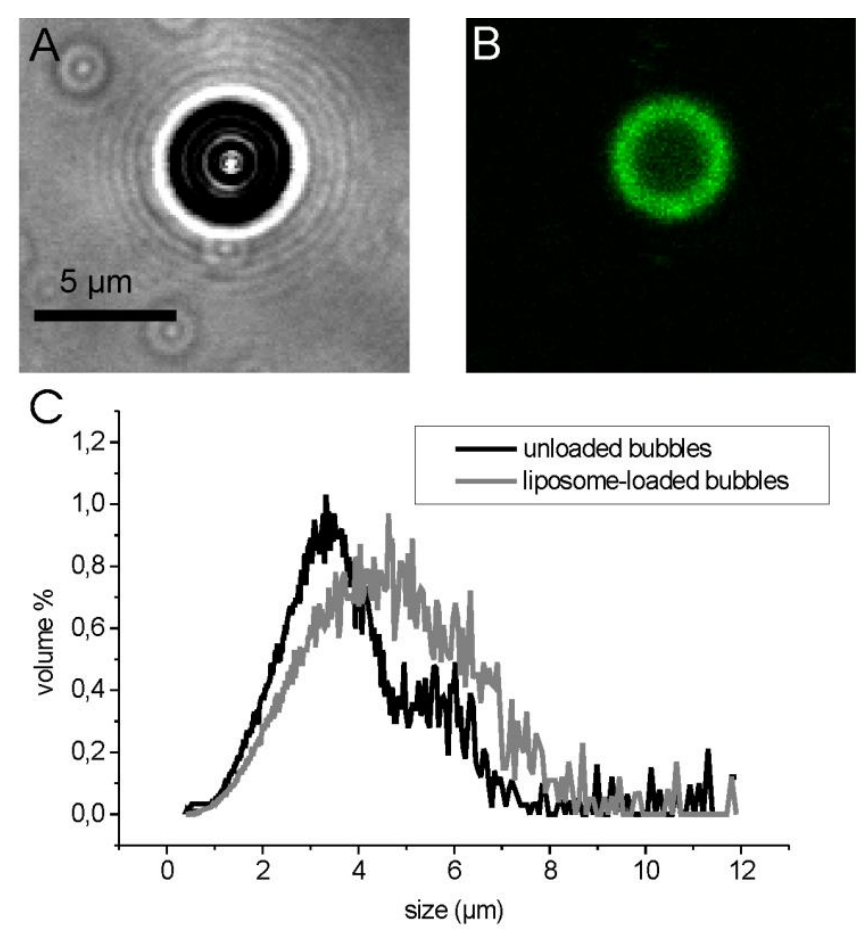

Figure 2: microscopy and Coulter Counter studies of liposome-loaded microbubbles: Transmission image (A) and confocal image (B) of a (bodipy-labeled) liposome-loaded microbubble. Size distributions of unloaded and liposome loaded microbubbles as obtained by Coulter Counter measurements (C) show an increase in mean size when liposomes are bound to the microbubbles' surface.

Subsequently we measured the size distribution and microbubble concentration of respectively unloaded and loaded microbubbles by Coulter Counter (Figure 2C). 
The unloaded microbubbles showed an average volume diameter of $3.6 \mu \mathrm{m}$ while the diameter of liposome-loaded microbubbles equaled $4.0 \mu \mathrm{m}$. The microbubble dispersions contained respectively $1.23 \times 10^{9}$ (unloaded) and $1.04 \times 10^{9}$ (loaded) microbubbles/ml. The $400 \mathrm{~nm}$ increase in average diameter of the bubbles upon loading with liposomes strongly indicates the formation of a single liposome layer at the microbubble surface as the hydrodynamic diameter of a single liposome is about $200 \mathrm{~nm}$.

\subsection{Improving the liposome-loading of the microbubbles}

Clearly, the more liposomes could be loaded onto the microbubble shell, the more drug could be released when the microbubble collapses. In a next step we evaluated whether the microbubble loading could be increased by using higher amounts of DSPE-PEG-SPDP lipids in the microbubble shell. We evaluated the amount of liposomes loaded per bubble through the use of bodipy-labeled liposomes and quantifying the fluorescence of the bodipy-liposome-loaded microbubbles through flow cytometry. Figure $3 \mathrm{~A}$ clearly shows that the mean fluorescence per bubble increases upon (a) adding more liposomes to the mixture and (b) using higher amounts of DSPE-PEG-SPDP in the lipid mixture. Figure 3B shows Coulter Counter measurements on the corresponding bubbles and clearly indicates that upon using 35\% DSPE-PEG-SPDP the microbubbles become substantially larger which explains the increased loading capacity of these microbubbles as more surface space per bubble is available. We noticed that it is possible to incorporate even higher amounts of DSPE-PEG-SPDP-lipids in the bubbles, however, the resulting microbubbles were unstable. Hence, we concluded that the use of a $35 \%$ molar ratio of DSPE-PEGSPDP-lipids is optimal for liposome loading. 

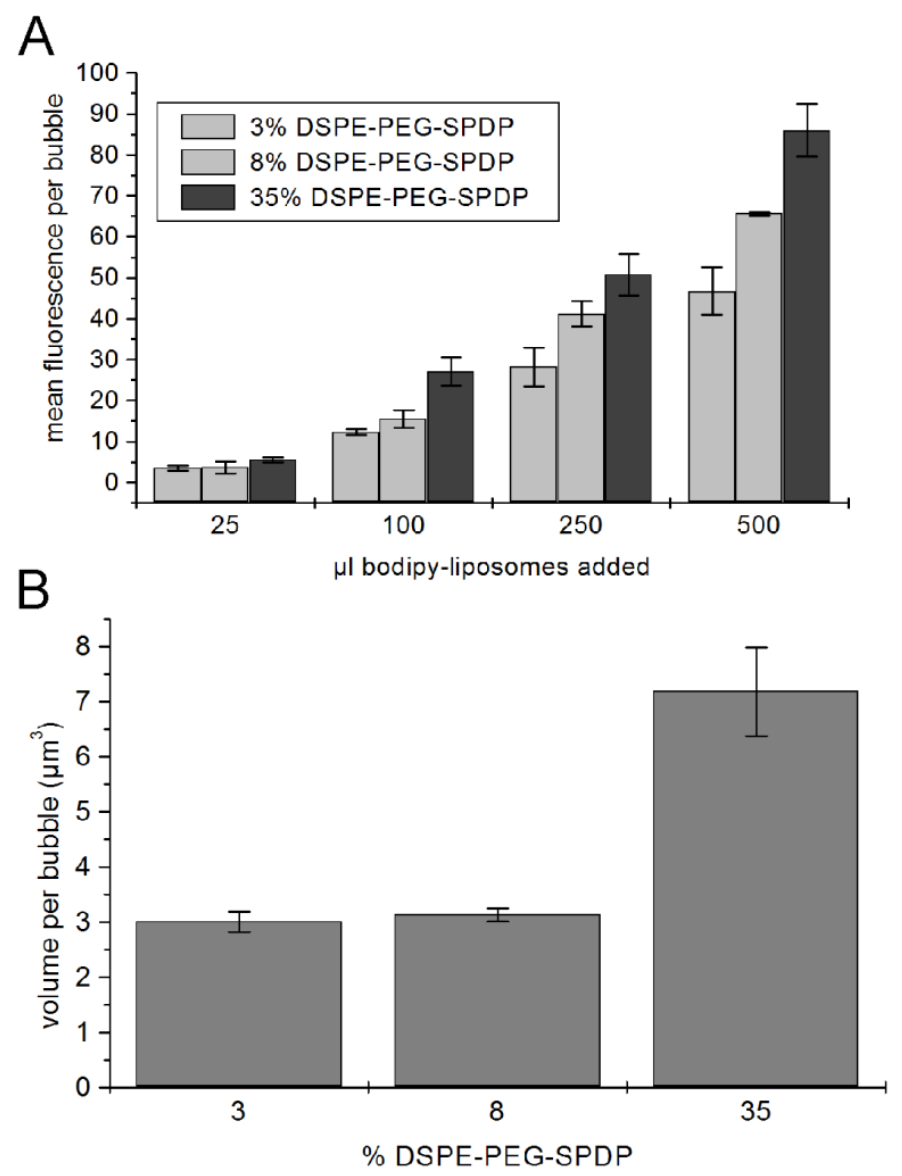

Figure 3: flow cytometry and coulter counter measurements of liposomeloading with increased PEGylation: (A) Results of flow cytometry on bodipyliposome loaded microbubbles: mean fluorescence per bubble in function of the amount of bodipy-liposomes added to the lipid mixture. (B) Volume per microbubble, as measured by Coulter Counter, in function of the amount of DSPE-PEG-SPDP used in the lipid mixture.

\subsection{Behavior of liposome-loaded microbubbles in plasma}

Clearly, successful ultrasound triggered drug release assumes that the microbubbles keep the drug as long as ultrasound energy is not applied. To judge whether liposome-loaded microbubbles remain stable once injected in plasma and do not release drug due to interactions with plasma components, we performed flow cytometry measurements on microbubbles loaded with bodipy-liposomes dispersed 
in human platelet poor plasma (Figure 4). We could not observe significant differences in the fluorescence of liposome-loaded microbubbles dispersed in respectively plasma and buffer indicating that liposome-loaded microbubbles remain stable in plasma. Hence we predict that the liposomes will remain bound to the bubbles after injection in the bloodstream, at least as long as they are not exposed to ultrasound.

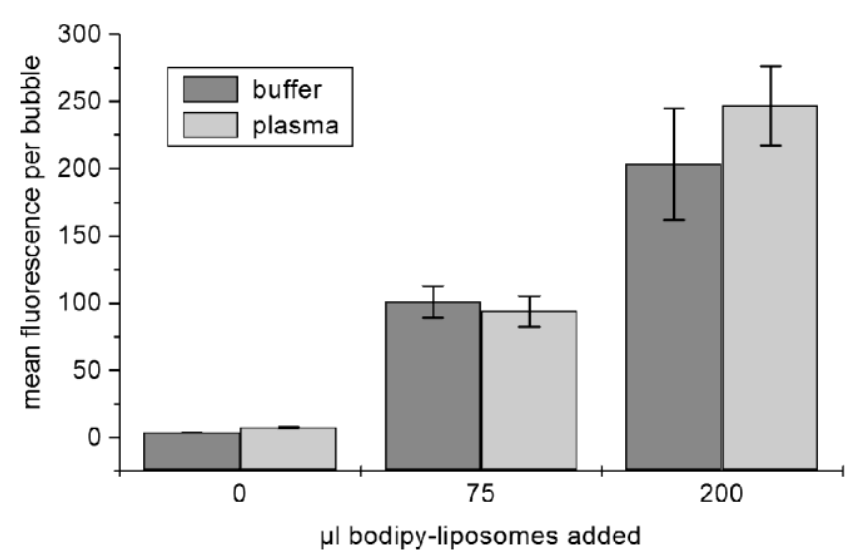

Figure 4: flow cytometry assessment of the stability of liposome-loading in plasma: Results of flow cytometry on bodipy-liposome-loaded microbubbles: mean fluorescence per bubble in function of the amount of bodipy-liposomes added to the lipid mixture.

\subsection{Aspecific interactions between the liposomes and the microbubbles}

Subsequently we evaluated whether liposomes can stick (i.e. become aspecifically bound) to the microbubbles. Therefore we prepared microbubbles with DSPE-MPEG lipids without functional end groups, thereby avoiding covalent binding. Figure $5 \mathrm{~A}$ shows the fluorescence of non-functionalized microbubbles exposed to bodipy-liposomes, as measured through flow cytometry. Clearly, liposomes bind aspecifically to the microbubbles. However, as could be expected, liposome loading 
of the microbubbles through covalent interactions enables significantly higher loading, which is of interest for use in drug delivery. One could speculate about the nature of the aspecific interactions between the liposomes and microbubbles. As schematically illustrated in Figure 5B, entanglements between PEG-chains on the liposomes and microbubbles may contribute to this phenomenon.

A

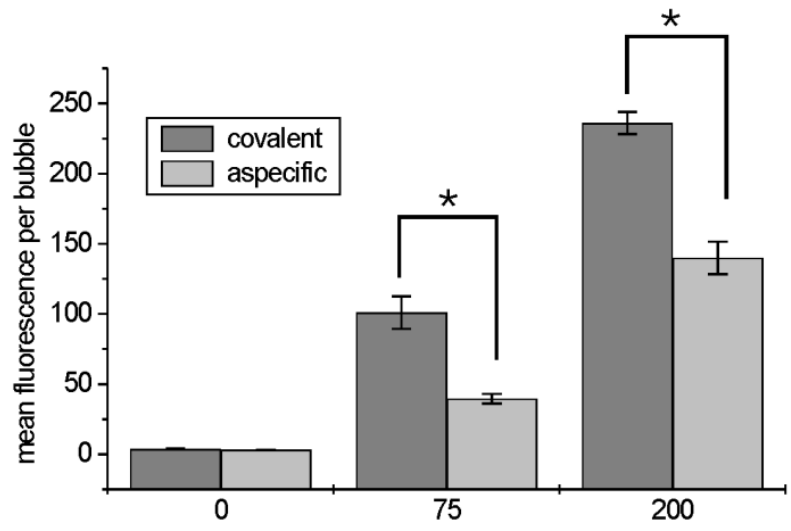

B

$\mu \mathrm{l}$ bodipy-liposomes added

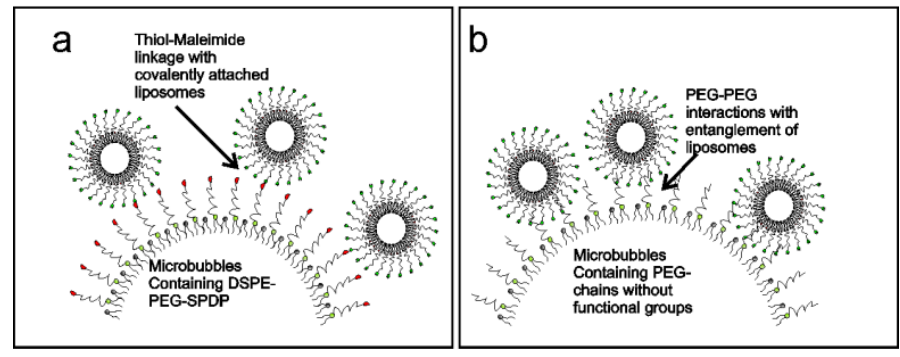

Figure 5: flow cytometry assessment of aspecific liposome-loading: (A) Results of flow cytometry on bodipy-liposome loaded microbubbles: mean fluorescence per bubble in function of the amount of bodipy-liposomes added to the lipid mixture. The micobubbles contained 35 mol\% DSPE-PEG-SPDP, the liposomes contained respectively $35 \%$ DSPE-mPEG or DSPE-PEG-MALEIMIDE. (B) Schematical depiction of specific (a) and aspecific (b) binding of the liposomes to the microbubbles through PEG-entanglements. 


\subsection{Coulter Counter measurements gain further insight in the amount of liposomes loaded}

Although flow cytometry experiments revealed clear information on the loading of the microbubbles with liposomes, it remained impossible to estimate the number of liposomes loaded per microbubble. This information is of interest as it defines the drug dose which can be loaded on the microbubbles' surface. We reasoned that measuring the increase in volume of a microbubble upon loading it with liposomes (defined as "liposome volume per bubble") in equation (2)) could give us valuable information. Dividing this "liposome volume per bubble" by the volume of one liposome allows us to estimate this number. Figure 6 shows the results of these experiments. For microbubbles with covalently attached liposomes we estimated that between 600 and 1300 liposomes can be loaded on the microbubbles' surface, which is significantly higher than the number which can be loaded through aspecific interactions. These data confirm the results obtained by Klibanov et al. [26] where is claimed that approximately 2000 liposomes with a diameter of $0.1 \mu \mathrm{m}$ can be bound to one bubble with a diameter of $2.5 \mu \mathrm{m}$. However the number of 600-1300 liposomes per bubble is based on a volumetric measurement with the Coulter Counter. Interestingly, the results obtained through Coulter Counter measurements confirm the data observed in Figure 5A which were obtained through flow cytometry. 


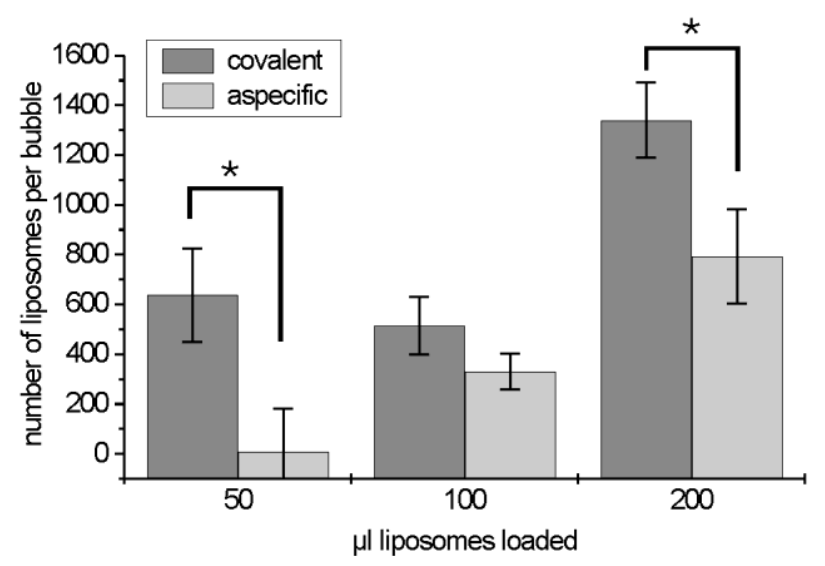

Figure 6: Estimation of the number of liposomes per bubble: Estimated number of liposomes per microbubble (containing 35 mol\% DSPE-PEG-SPDP) as measured by Coulter Counter.

\subsection{Ultrasound induced DOX release from DOX-liposome-loaded microbubbles}

Next we evaluated whether applying ultrasound on the DOX-liposome microbubble results in the release of DOX from the liposomes. As DOX encapsulated in liposomes has a lower fluorescence intensity than the corresponding amount of freely dissolved DOX, an increase in fluorescence could be expected upon applying ultrasound to the DOX-liposome loaded microbubbles. Figure 7 shows a significant increase in fluorescence when liposome-loaded microbubbles are subjected to ultrasound, which indicates that free doxorubicin is released from the liposomes after insonation. 


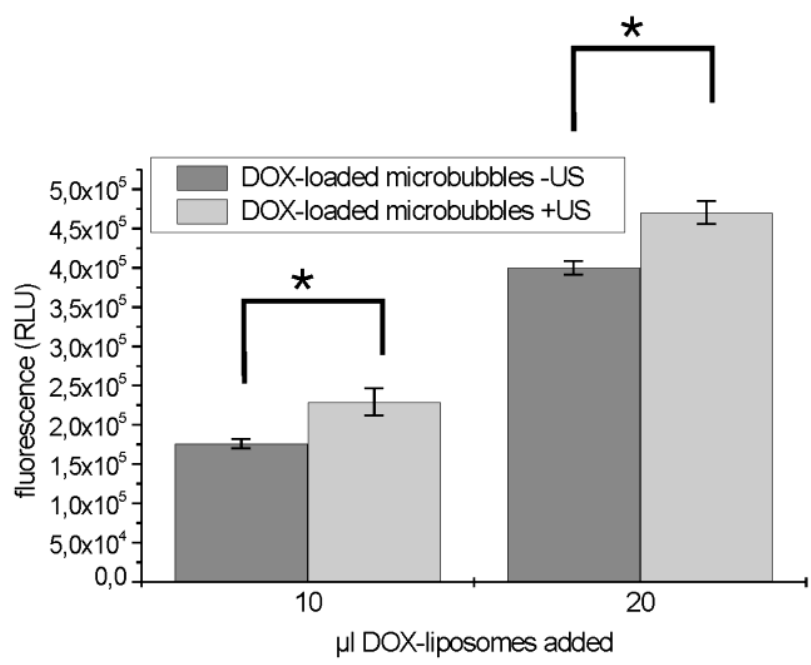

Figure 7: Fluorimetric analysis of ultrasound induced free DOX release:

Fluorescence intensity of DOX (expressed as relative light units $(R L U)$ ) significantly increases when liposome loaded microbubbles are subjected to ultrasound, indicating that DOX leaks out of destroyed liposomes after ultrasound treatment.

\subsection{Biological efficacy of DOX-loaded liposome bubbles treated with ultrasound}

In a next step we evaluated the tumor cell killing efficacy of DOX-liposome loaded microbubbles. Figure 8B shows the results of an in vitro cytotoxicity assay using different cell killing strategies. Clearly, ultrasound treatment of DOX-liposome loaded microbubbles (white bars) results in a significantly stronger killing of the cells. The $x$-axes in Figure 8B indicates the concentration of DOX in the Opticells ${ }^{\mathrm{TM}}$. Note that a higher DOX concentration was obtained through the use of microbubbles which were more loaded with DOX-liposomes (i.e. the number of DOX-liposome loaded microbubbles per Opticell ${ }^{\mathrm{TM}}$ was constant). Clearly, the 'heavier' the microbubbles are loaded with DOX-liposomes the more cytotoxic they are. Importantly, without ultrasound DOX-liposome loaded microbubbles do not kill cells (dark gray bars in Figure 8B). As the DOX-liposome loaded microbubbles are not 
cytotoxic as long as they are not subjected to ultrasound, we suggest that such bubbles in combination with ultrasound may allow targeted release of DOX. We would like to note that, as the cytotoxicity experiments were done on a cell monolayer, cells were occasionally (physically) removed from this layer due to the ultrasound energy or the microbubbles treatment itself. This could induce false positive results in the cytotoxity experiments, although microbubble concentrations are used that induced a minimal cell displacement as verified microscopy (data not shown). The bars in Figure 8A show that at microbubble concentrations used in the experiments there was no significant killing of the cells due to the microbubble destruction itself; the observed cytotoxicity was similar to that observed with the blank (optimem).

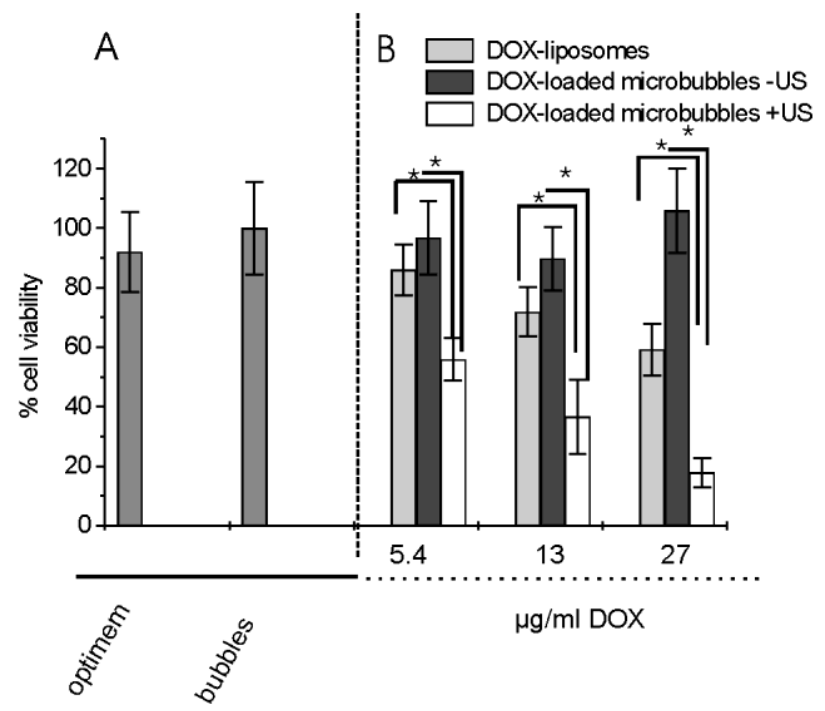

Figure 8: Viability measurements on melanoma cells: $(A)$ Effect of microbubbles alone in presence of ultrasound on melanoma cell viability compared to no treatment (optimem). (B) Viability of melanoma cells after treatment with DOX-liposome loaded microbubbles, with ultrasound treatment (light gray bars), DOX-liposome loaded microbubbles without ultrasound treatment (dark gray bars) and DOX-liposomes 
alone, were measured using augmenting amounts of DOX-liposomes in an Opticell ${ }^{\mathrm{TM}}$ plate.

\subsection{Can dose reduction be achieved with DOX-liposome loaded microbubbles?}

In the experiments in Figure 8 the DOX-liposome loaded microbubble dispersions did still contain an amount of free DOX-liposomes as it was experimentally difficult to separate free liposomes from the microbubbles. We were especially interested in the cell killing properties of the DOX-lipsosome loaded microbubbles themselves. We described above that between 600 and 1300 liposomes can be bound per microbubble. Therefore we prepared a DOX-liposome microbubble dispersion using an amount of DOX-liposomes which is expected to become fully loaded on the microbubbles. One can calculate that, when the microbubbles are loaded with this amount of liposomes, approximately $5 \mu \mathrm{g}$ of DOX is loaded on the microbbubbles present in a $1 \mathrm{~mL}$ dispersion. Under these conditions we performed the cytotoxicity measurements, which results in a DOX-concentration of $0.5 \mu \mathrm{g} / \mathrm{ml}$ in the Opticells ${ }^{\mathrm{TM}}$ after applying the DOX-liposome microbubble dispersion to the cells. Figure 9 compares the cell killing at a DOX concentration of $0.5 \mu \mathrm{g} / \mathrm{ml}$ in the Opticells ${ }^{\mathrm{TM}}$, respectively when free DOX and DOX-liposome microbubble dispersion (without free DOX-liposomes) were applied. Despite the very low DOX-concentration in the Opticells ${ }^{\mathrm{TM}}$, DOX-liposome loaded microbubbles were clearly cytotoxic (in case ultrasound was applied). In contrast, free DOX did not cause any significant tumor cell death. Our results indicate that very low amounts of doxorubicin can become significantly effective when loaded onto microbubbles and 
exposed to ultrasound. An interesting observation towards the delivery of DOX as a lower dose may become sufficient in the treatment of patients.

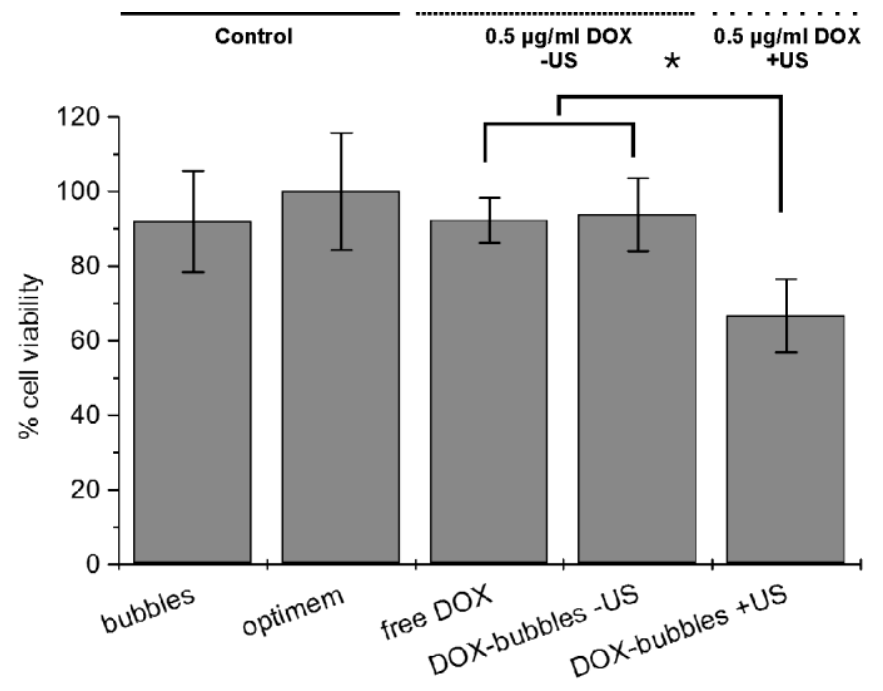

Figure 9: Viability measurements on melanoma cells with reduced dose: Viability of melanoma cells after exposure to respectively DOX-liposome loaded microbubbles (with and without ultrasound (US) treatment) and free DOX. The DOXconcentration in the wells was $0.5 \mu \mathrm{g} / \mathrm{ml}$. Note that in these experiments we did not expect free DOX-liposomes to be present in the DOX-liposome loaded microbubble dispersion.

\section{DISCUSSION}

In this paper we show that it is possible to design a safe and simple system for ultrasound guided drug delivery and we show that this system is very efficient in vitro, even when low amounts of model drug (DOX) are used. However, these in vitro experiments were performed in an ideal setting, because microbubbles were contacting the melanoma cells while sonicated, which probably leads to a more efficient sonoporation. In this respect, one could speculate on how to apply this system in an in vivo setting. Since microbubbles are relatively large particles, they will 
not pass the endothelial barrier in the tumor tissue, hence drug release has to occur in the blood vessels. The possible future clinical application of this system, will depend on how efficient the drug will penetrate the tissue under ultrasound application. Fortunately it has been recently shown that ultrasound and microbubbles can lead to a permeabilization of the endothelial barrier in (tumor) tissue which causes extravasation of coinjected molecules/drugs [27], other studies prove that ultrasound and microbubbles can even cause a local opening of the Blood Brain Barrier (BBB) [28]. In our measurements we have clearly shown that active compound is released out of the liposomes after implosion of the microbubbles, this release of free DOX can diffuse into the tissue after disruption of the endothelial barrier and can have a fast effect on the tumor cells. Furthermore, it is likely that not every liposome is destructed after ultrasound insonation, but is released in the surrounding environment. These liposomes can also be delivered into the tissue after endothelial barrier disruption. Another mechanism of tumor killing in vivo can be the specific killing of the endothelial cell layer directly contacting the microbubbles that are affected by the ultrasound. This mechanism is directly comparable with the in vitro experiments performed in this paper. The specific killing of endothelial cells will impair blood flow in the tumor and induce necrosis of the tumor. To even improve contact between these endothelial cells and the liposome-loaded microbubble a targeting ligand might be introduced in the system. The use of liposome-loaded microbubbles will in the end allow killing of tumor cells using less drug, because the drug will only be delivered where and when ultrasound is applied. Furthermore, future in vivo experiments with this system will have to answer the question whether the efficiency of the therapy will be improved too, if sonoporation allows direct delivery of the drug in endothelial cells and increased uptake in tumor cells. 
Another issue which is still prone to discussion is the drug dose which can be maximally loaded on and thus delivered by the microbubbles. A microbubble is relatively small, which limits the space for drug loading. We believe that binding (drug containing) vesicles (like liposomes) on the surface of the bubbles maximizes the amount of drug that can be loaded. From this point of view this strategy may be an advantage when compared with other approaches reported to load microbubbles with drugs, like e.g. ones which load the drug into an inner oil layer or in the bubble shell itself $[17,18]$. Though we showed that approximately 600 to 1300 liposomes can be bound per single microbubble the amount of DOX which becomes loaded in this way still remains rather limited. The loading may be further improved by applying multiple layers of liposomes around the microbubbles. The materials reported here can only be further clinically evaluated provided one can overcome the low drug loading obstacle. Nowadays cancer patients are treated with a relatively high dose of DOXYL ${ }^{\mathrm{TM}}$, i.e. $40-50 \mathrm{mg}$ DOX per injection. Designing drug-loaded microbubbles which would allow such a high DOX dosing is highly likely impossible unless really high amounts of bubbles (in the order of $1 \times 10^{13}$ bubbles $/ \mathrm{ml}$ ) could be injected. However, considering the targeted and more efficient delivery of DOX when ultrasound and DOX-liposome loaded bubbles are used, we believe that such high DOX-doses might be no longer necessary, which may also reduce the severe sideeffects of DOX-therapy in particular and chemotherapy in general.

Since apparati suitable for in vivo ultrasound assisted drug delivery that are able to deliver ultrasound into a focal spot deep into the tissue are allready under full development [29], the clinical evaluation of the drug-loaded microbubbles reported in this paper will smoothly move on and hopefully make it from bench to bedside. 


\section{CONCLUSIONS}

This paper showed that that DOX-liposome loaded microbubbles can be obtainbed through self-assembly of (functionalized) phospholipids, drug-loaded liposomes and perfluorobutane gas. Importantly, this single step process results in a material which meets all criteria for clinical applicability: (a) no immunogenic compounds are used; (b) sterilization can easily be achieved through straightforward techniques and (c) adequate amounts of liposomes can be stabily loaded on the surface of the bubbles. Through flow cytometry and Coulter Counter measurements we showed a maximal loading of the microbubbles with liposomes when the lipid shell of the microbubbles was composed of $35 \mathrm{~mol} \%$ of DSPE-PEG-SPDP, resulting in approximately 600 to 1300 liposomes bound per single microbubble. Importantly, the liposomes remained bound to the mirobubbles' surface upon incubation in plasma, being a clear requirement for clinical application. Ultrasound induced microbubble collapse clearly induced the release of DOX. When compared with free DOX and DOX-liposomes, we observed that DOX-liposome loaded microbubbles in combination with ultrasound showed a significantly stronger killing of cancer cells. It is our opinion that the DOXliposome loaded microbubbles described in this paper provide an opportunity for ultrasound targeted cancer therapy.

\section{ACKNOWLEDGEMENTS}

This research was funded by the EU FP7 collaborative projects SONODRUGS (NMP-4-LA-2008-213706) and ARISE, and the FWO-flanders research project G.0187.11. Ine Lentacker is a post-doctoral fellow of the FWO flanders the financial support of this institute is thanked. Luis Diaz Gomez and Dieter Uyttersprot are thanked for technical support. 


\section{REFERENCE LIST}

[1] A. Bouakaz, M. Versluis, J.N. de, High-speed optical observations of contrast agent destruction, Ultrasound Med. Biol. 31 (2005) 391-399.

[2] J. Chomas, P. Dayton, D. May, K. Ferrara, Nondestructive subharmonic imaging, IEEE Trans. Ultrason. Ferroelectr. Freq. Control 49 (2002) 883-892.

[3] M. Postema, W.A. van, C.T. Lancee, J.N. de, Ultrasound-induced encapsulated microbubble phenomena, Ultrasound Med. Biol. 30 (2004) 827-840.

[4] C.M. Newman, T. Bettinger, Gene therapy progress and prospects: ultrasound for gene transfer, Gene Ther. 14 (2007) 465-475.

[5] K. Ferrara, R. Pollard, M. Borden, Ultrasound microbubble contrast agents: fundamentals and application to gene and drug delivery, Annu. Rev. Biomed. Eng 9 (2007) 415-447.

[6] S. Mehier-Humbert, T. Bettinger, F. Yan, R.H. Guy, Plasma membrane poration induced by ultrasound exposure: implication for drug delivery, J. Control Release 104 (2005) 213-222.

[7] C.D. Ohl, M. Arora, R. Ikink, J.N. de, M. Versluis, M. Delius, D. Lohse, Sonoporation from jetting cavitation bubbles, Biophys. J. 91 (2006) 4285-4295.

[8] M.M. Forbes, R.L. Steinberg, W.D. O'Brien, Jr., Examination of inertial cavitation of Optison in producing sonoporation of chinese hamster ovary cells, Ultrasound Med. Biol. 34 (2008) 2009-2018.

[9] A. Rahim, S.L. Taylor, N.L. Bush, G.R. ter Haar, J.C. Bamber, C.D. Porter, Physical parameters affecting ultrasound/microbubble-mediated gene delivery efficiency in vitro, Ultrasound Med. Biol. 32 (2006) 1269-1279.

[10] B.D. Meijering, L.J. Juffermans, W.A. van, R.H. Henning, I.S. Zuhorn, M. Emmer, A.M. Versteilen, W.J. Paulus, W.H. van Gilst, K. Kooiman, J.N. de, R.J. Musters, L.E. Deelman, O. Kamp, Ultrasound and microbubble-targeted delivery of macromolecules is regulated by induction of endocytosis and pore formation, Circ. Res. 104 (2009) 679-687.

[11] A. Yudina, M. Lepetit-Coiffe, C.T. Moonen, Evaluation of the Temporal Window for Drug Delivery Following Ultrasound-Mediated Membrane Permeability Enhancement, Mol. Imaging Biol. (2010)

[12] S. Meairs, A. Alonso, M. Fatar, R. Kern, M. Hennerici, Microbubbles traversing the blood-brain barrier for imaging and therapy, Med. Biol. Eng Comput. 47 (2009) 839-849. 
[13] L.H. Treat, N. McDannold, N. Vykhodtseva, Y. Zhang, K. Tam, K. Hynynen, Targeted delivery of doxorubicin to the rat brain at therapeutic levels using MRIguided focused ultrasound, Int. J. Cancer 121 (2007) 901-907.

[14] Z. Gao, A.M. Kennedy, D.A. Christensen, N.Y. Rapoport, Drug-loaded nano/microbubbles for combining ultrasonography and targeted chemotherapy, Ultrasonics 48 (2008) 260-270.

[15] S. Hernot, A.L. Klibanov, Microbubbles in ultrasound-triggered drug and gene delivery, Adv. Drug Deliv. Rev. 60 (2008) 1153-1166.

[16] I. Lentacker, B.G. De Geest, R.E. Vandenbroucke, L. Peeters, J. Demeester, S.C. De Smedt, N.N. Sanders, Ultrasound-responsive polymer-coated microbubbles that bind and protect DNA, Langmuir 22 (2006) 7273-7278.

[17] S. Tinkov, G. Winter, C. Coester, R. Bekeredjian, New doxorubicin-loaded phospholipid microbubbles for targeted tumor therapy: Part I - Formulation development and in-vitro characterization, J. Control Release (2010)

[18] K. Kooiman, M.R. Bohmer, M. Emmer, H.J. Vos, C. Chlon, W.T. Shi, C.S. Hall, S.H. de Winter, K. Schroen, M. Versluis, J.N. de, W.A. van, Oil-filled polymer microcapsules for ultrasound-mediated delivery of lipophilic drugs, J. Control Release 133 (2009) 109-118.

[19] M.R. Bohmer, A.L. Klibanov, K. Tiemann, C.S. Hall, H. Gruell, O.C. Steinbach, Ultrasound triggered image-guided drug delivery, Eur. J. Radiol. 70 (2009) 242253.

[20] I. Lentacker, S.C. De Smedt, N.N. Sanders, Drug loaded microbubble design for ultrasound triggered delivery, Soft Matter 5 (2009) 2161-2170.

[21] I. Lentacker, B. Geers, J. Demeester, S.C. De Smedt, N.N. Sanders, Design and evaluation of doxorubicin-containing microbubbles for ultrasound-triggered doxorubicin delivery: cytotoxicity and mechanisms involved, Mol. Ther. 18 (2010) 101-108.

[22] I. Lentacker, S.C. De Smedt, J. Demeester, V. Van Marck, M. Bracke, N.N. Sanders, Lipoplex-loaded microbubbles for gene delivery: A Trojan horse controlled by ultrasound, Advanced Functional Materials 17 (2007) 1910-1916.

[23] R.E. Vandenbroucke, I. Lentacker, J. Demeester, S.C. De Smedt, N.N. Sanders, Ultrasound assisted siRNA delivery using PEG-siPlex loaded microbubbles, J. Control Release 126 (2008) 265-273.

[24] I. Lentacker, R.E. Vandenbroucke, B. Lucas, J. Demeester, S.C. De Smedt, N.N. Sanders, New strategies for nucleic acid delivery to conquer cellular and nuclear membranes, J. Control Release 132 (2008) 279-288.

[25] C. Brancewicz, D.H. Rasmussen, B. Papahadjoulos-Sternberg, Hydrophobic gas bubble formation in definity $(R)$ : A freeze fracture electron microscopy study, Journal of Dispersion Science and Technology 27 (2006) 761-765. 
[26] A.L. Klibanov, T.I. Shevchenko, B.I. Raju, R. Seip, C.T. Chin, Ultrasoundtriggered release of materials entrapped in microbubble-liposome constructs: A tool for targeted drug delivery, J. Control Release (2010)

[27] M.R. Bohmer, C.H. Chlon, B.I. Raju, C.T. Chin, T. Shevchenko, A.L. Klibanov, Focused ultrasound and microbubbles for enhanced extravasation, J. Control Release (2010)

[28] K. Hynynen, Ultrasound for drug and gene delivery to the brain, Adv. Drug Deliv. Rev. 60 (2008) 1209-1217.

[29] R. Seip, C.T. Chin, C.S. Hall, B.I. Raju, A. Ghanem, K. Tiemann, Targeted ultrasound-mediated delivery of nanoparticles: on the development of a new HIFU-based therapy and imaging device, IEEE Trans. Biomed. Eng 57 (2010) 61-70. 